\title{
Candida albicans and Candida dubliniensis in Periodontitis in Adolescents and Young Adults
}

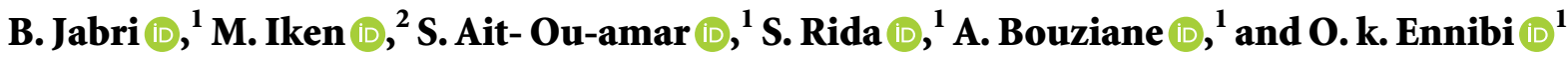 \\ ${ }^{1}$ Research Laboratory in Oral Biology and Biotechnology, Faculty of Dental Medicine, Mohammed V University in Rabat, \\ Rabat, Morocco \\ ${ }^{2}$ Clinical Biology Department, Faculty of Medicine and Pharmacy, Mohammed V University in Rabat, Rabat, Morocco
}

Correspondence should be addressed to O. k. Ennibi; o.ennibi@um5s.net.ma

Received 9 October 2021; Accepted 27 December 2021; Published 11 January 2022

Academic Editor: Chioma Blaise Chikere

Copyright ( $\odot 2022$ B. Jabri et al. This is an open access article distributed under the Creative Commons Attribution License, which permits unrestricted use, distribution, and reproduction in any medium, provided the original work is properly cited.

\begin{abstract}
Aim. This study aims to evaluate the association of Candida albicans and Candida dubliniensis with periodontitis in adolescents and young adults in a Moroccan population. Methods. 426 subjects aged between 12 and 25 years were recruited for the study. A pool of plaque sample was taken. Samples were cultured on Sabouraud Chloramphenicol medium at $37^{\circ} \mathrm{C}$ for $24-48$ hours and then identified by the Vitek 2 YST system. Clinical data and presence of Candida albicans and Candida dubliniensis were analyzed using Jamovi (Version 1.8). Results. Candida albicans was observed in 25 subjects among 68 diseased patients (37\%) and in 60 subjects among 358 healthy patients (17\%). It can be reported that under normal yeast conditions, there is a statistically significant difference between these two groups $(P<0.001)$. Candida dubliniensis was more prevalent in periodontitis than in healthy subjects $(P=0.026)$. Regarding clinical variables, subgroups of periodontitis subjects showed significant statistical differences for periodontal probing depth, clinical attachment loss, and number of decayed teeth in advanced periodontitis in comparison with initial or mild periodontitis. The results also indicate that the presence of the two species of Candida is not related to gender or age $(P>0.05)$ nor related to the severity of the periodontal disease in this population. Conclusion. Within the limits of our study, Candida albicans is more frequently associated with periodontitis. The potential role of C. albicans in periodontitis pathogenesis is very complex. More studies on biofilm associated with different forms of periodontitis are necessary. It is also important to assess the coexistence of periodontitis and caries and the associated biofilms.
\end{abstract}

\section{Introduction}

Periodontal diseases are among the most common infectious diseases affecting the oral cavity. They are associated with the presence of microbial biofilm that affects the immune-inflammatory response. The unbalance between biofilm accumulation and host response causes the destruction of the periodontium, which can lead to the possible loss of teeth $[1,2]$. The periodontal pocket is an elementary lesion of periodontitis and serves as reservoirs of microbial agents [3]. Microbiota associated with periodontitis is very complex and play a major role in the development and the severity of the disease. The associated microorganisms with periodontitis are both pathogenic and commensally species having a pathogenicity favored by environmental factors [4].
Several studies have reported that periodontal diseases are caused by highly virulent bacteria, that is, Actinobacillus actinomycetemcomitans $(\mathrm{Aa})$ and Porphyromonas gingivalis $(P g)$ [5]. However, the role of other microorganisms such as yeasts is poorly known. Some data showed a prevalence of yeast up to 10 to $30 \%$ in periodontal pockets of healthy subjects $[6,7]$.

The most frequently isolated yeast from the oral cavity of healthy individuals is Candida with frequency of 31 to 55\% [8]. Several species of this Candida genus have been identified in periodontal pockets from 7.1 to $19.6 \%$ of patients with periodontal disease $[9,10]$. The most frequently associated with these diseases is Candida albicans yeast. Other less widespread species have been isolated too $[11,12]$, such as Candida dubliniensis, a yeast closely phylogenetically 
related to Candida albicans, with differences in genome sequencing [13].

This study aims to assess the association of Candida albicans and Candida dubliniensis with periodontitis among teenagers and young adults in a Moroccan population.

\section{Materials and Methods}

2.1. Study Population. The target population for this crosssectional study was teenagers and young adults. The subjects were students from high schools and faculties randomly selected in the city of Rabat, Morocco.

Based on a frequency of Candida of $3.57 \%$ in healthy group and $26 \%$ in subjects with periodontitis (Uzura et al. (2008), a power of $95 \%$, a risk $\alpha$ of $1 \%$, and a ratio of cases to controls $1: 5$ ), the sample size estimation was 57 subjects in periodontitis group and 287 individuals in healthy group (a total of 344 subjects). We took into account the possible dropout cases in our study, so the sample size had been increased by $20 \%$. Therefore, the global sample estimation was 413 subjects.

The research was done in accordance with the Ethical Principles for Medical Research involving Human Subjects outlined in the Declaration of Helsinki, and approval was obtained from the Biomedical Research Ethics Committee (CERB) of the Faculty of Medicine and Pharmacy of the Mohamed V University in Rabat under the number 102/19. We also asked for an authorization to access schools and faculties (authorization numbers: 18/8164; 19/0629; 19/ 6113).

All subjects were informed about the study and agreed to participate. The consents were signed by the students aging or above 18 years old and by the parents of those who were under 18.

Excluded students were those with infectious diseases, patients who had received periodontal treatment or had used an antibiotic during the last three months prior the plaque sampling, patients who had orthodontic treatment, and those who used tobacco or having diabetes.

\subsection{Methods}

2.2.1. Periodontal Examination. Prior to the clinical examination, consenting students were interviewed using a structured questionnaire to collect demographic and behavioral data.

To assess the periodontal status, the following clinical variables were used: O'Leary plaque index (PI), bleeding on probing (BoP), full mouth periodontal probing (FMPP), and clinical attachment loss (CAL). Periodontal probing and attachment loss measurements were recorded at six sites per tooth, except third molars, using a North Carolina periodontal probe (Hu-Friedy, Chicago, IL, USA).

Based on the clinical examination, a subject was assigned a status of periodontitis or nonperiodontitis subject according to the 2017 World Workshop Classification of Periodontal Diseases and Conditions [14]. Periodontitis is defined as the presence of clinical attachment loss in at least 2 nonadjacent teeth or the presence of buccal or oral clinical attachment loss $\geq 3 \mathrm{~mm}$ with pocketing $>3 \mathrm{~mm}$ in at least 2 teeth [15]. To address measurement errors, the threshold of CAL was set at $2 \mathrm{~mm}$.

Moreover, stages and grades were also defined based on the severity and complexity of periodontal breakdown and based on the rate of periodontitis progression, respectively. For stages, the following criteria were used: Stage I, initial periodontitis where CAL at the site of greatest loss was 1 to $2 \mathrm{~mm}$, there was no tooth loss due to periodontitis, and maximum probing depth was $4 \mathrm{~mm}$; Stage II, moderate periodontitis in which CAL at the site of greatest loss was 3 to $4 \mathrm{~mm}$, there was no tooth loss due to periodontitis, and maximum probing depth was $5 \mathrm{~mm}$; Stage III, severe periodontitis with CAL at the site of greatest loss was $\geq 5 \mathrm{~mm}$, probing depth was $\geq 6 \mathrm{~mm}$, furcation involvement was Class II or III, tooth loss due to periodontitis was $\leq 4$ teeth, and there was a risk of potential additional tooth loss; Stage IV, advanced periodontitis with CAL at the site of greatest loss was $\geq 5 \mathrm{~mm}$, tooth loss due to periodontitis was $\geq 5$ teeth, and there was risk of with extensive tooth loss and potential for loss of dentition.

For progression of periodontitis, grading was based on the estimation of importance of longitudinal data (CAL) as follows: Grade B, having low loss of attachment and periodontal destruction that commensurate with biofilm deposits; Grade C, CAL and periodontal destruction exceeds expectation given biofilm deposits; and specific clinical features evocative of periods of rapid progression and/or early onset disease (e.g., molar/incisor pattern).

Risk factors (smoking and diabetes) that may modify the grade were considered as exclusion criteria for this study.

The presence of Candida albicans was assessed in nonperiodontitis and periodontitis patients and in different subgroups of periodontitis subjects.

2.2.2. Mycological Sampling. A pool of subgingival biofilm was randomized at six sites per patient, using an absorbent paper point inserted into the periodontal pocket or sulcus. The subgingival samples were transferred into an Eppendorf tube containing $2 \mathrm{ml}$ of PBS (sodium chloride $137.0 \mathrm{mM} \mathrm{NaCl}$, disodium phosphate $10.0 \mathrm{mM}$ Na2HPO4, monopotassium phosphate $2.0 \mathrm{mM} \mathrm{KH} 2 \mathrm{PO}$, and potassium chloride $2.7 \mathrm{mM}$ $\mathrm{KCl}$ ). The samples were then transported immediately to the laboratory for analysis. They were processed for yeast identification. Microbial culture and identification tests were performed at the Research Laboratory of Oral Biology and Biotechnology (LRBOB) Faculty of Dental medicine in Rabat, and the Parasitology-Mycology Laboratory of the Mohamed V Military Teaching Hospital, Rabat, Morocco.

2.2.3. Identification of Candida. $100 \mu \mathrm{l}$ of each pooled sample was aseptically inoculated into Petri dishes containing Sabouraud Chloramphenicol medium and incubated at $37^{\circ} \mathrm{C}$ for 24 to 48 hours [16]. The Candida colonies on this medium are creamy whitish and smooth (Figure 1) [17]. Under direct examination of the culture, the colonies appear as oval, ovoid, or elongated and possibly like budding elements (Figure 2) [18]. 


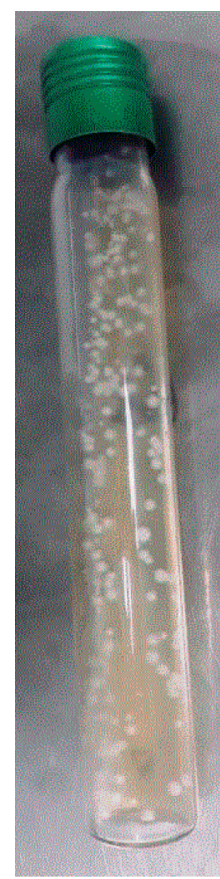

FIGURE 1: Macroscopic examination of the appearance of Candida on Sabouraud Chloramphenicol culture medium.

The colonies of Candida genus in each sample were numbered using the $\mathrm{CFU} / \mathrm{mL}$ (colony forming units).

Identification by the Vitek 2 YST card system is as follows.

The Vitek 2 Yeast card was used according to the manufacturer's instructions by the Vitek 2 system (YST Insert Package: Ref 21343, Biomérieux, Inc.).

After a culture of 24 to 48 hours, the manipulation is minimized in a simple step of preparation, standardization, and dilution of the inoculum (Mc Farland: 1.80-2.20). The following strain, Candida albicans ATCC 90028, was used as a reference strain.

The cards, consisting of 64 wells with 47 biochemical tests, are automatically integrated, filled, sealed, and incubated at a temperature of $35^{\circ} \mathrm{C}$.

The efficiency of the Vitek 2 Compact system is based on a colorimetric technique that is based on the reading of the cards every 15 minutes on the basis of three different wavelengths.

The time to obtain results of identification of yeasts is 18 hours.

2.2.4. Data Analysis. Data analysis was conducted using Jamovi (Version 1.8) (The Jamovi Project, Sidney, Australia). Descriptive data were presented as a mean $( \pm S D)$ and median and quartiles for quantitative measures and as a percentage for qualitative values.

Differences of clinical variables and Candida albicans and Candida dubliniensis status between nonperiodontitis patient and periodontitis subjects and between the subgroups of periodontitis groups were analyzed by parametric and nonparametric tests for variables expressed as median and quartiles.

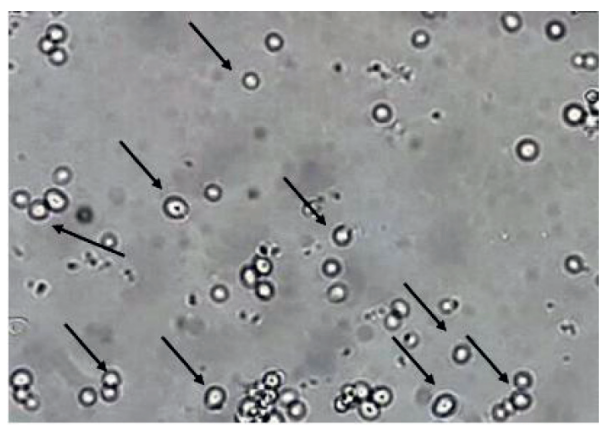

Figure 2: Appearance of Candida in a fresh state.

The threshold of statistical significance retained was $P<0.05$.

\section{Results}

A total of 426 students participated in the study. They were aged between 12 and 25 years old from both genders. The mean age of the population was $18,19 \pm 3,20$. The study population was divided mainly onto 2 groups: periodontitis subjects and nonperiodontitis subjects. Among 426 subjects, $16 \%$ had periodontitis. Demographic and clinical data of the population are summarized in Table 1.

The study population had globally very high plaque index and bleeding on probing index, even if the difference was statically significant between nonperiodontitis subjects and periodontitis subjects (Table 1).

Candida dubliniensis represented $4 \%$ of Candida genus in the cultivated samples, whereas Candida albicans represented $20 \%$.

The comparison of the presence of $C$. albicans and C. dubliniensis regarding age and gender did not show significant statistical differences $(P>0.05)$ (Table 2).

When comparing periodontitis subgroups, accordingly to severity and rate of progression (stages and grades), there was no statistical differences for age $(P>0.05)$ (Tables 3 and 4$)$.

Regarding clinical variables, subgroups of periodontitis subjects showed significant statistical differences for periodontal probing depth, clinical attachment loss, and number of decayed teeth in periodontitis Stage III and grade C in comparison with periodontitis Stage I or II and Grade B, respectively (Tables 3 and 4 ). The results also indicate that the presence of the two species of Candida is not related to the severity of the periodontal disease in this population (Tables 3 and 4).

\section{Discussion}

The current study found that $16 \%$ of the studied population aged between 12 and 25 years old had periodontitis. All over the world, studies suggested that the prevalence of periodontitis is $15-30 \%$ in adults, and sometimes higher [19-21]; among African adolescents, the prevalence of aggressive periodontitis was estimated as $3.4 \%$ to $6.5 \%$ [22, 23].

In a Moroccan population, Kissa et al. in 2016 found a prevalence of periodontitis of $11.3 \%$ (94/830) in students aged 12 to 25 [24]. However, in that study, the threshold of 
TABLE 1: Demographic and clinical characteristics and the presence of yeast in the nonperiodontitis and periodontitis groups.

\begin{tabular}{|c|c|c|c|}
\hline & Nonperiodontitis subjects, $N=358(84 \%)$ & Periodontitis subjects, $N=68(16 \%)$ & $P$ value \\
\hline Gender (male/female) & $198 / 16055 \% / 45 \%$ & $36 / 3253 \% / 47 \%$ & $0.719^{(a)}$ \\
\hline Age (years) $($ mean $\pm S D)$ & $17.81 \pm 3.20[12-24]$ & $20.22 \pm 2.31[14-25]$ & $<0.001^{(\mathrm{b})}$ \\
\hline Plaque index $(\%)$ & $100[97-100]$ & $100[100-100]$ & $0.001^{(\mathrm{c})}$ \\
\hline Bleeding on probing (BOP) $(\%)$ & $86[38-100]$ & $100[100-100]$ & $<0.001^{(\mathrm{c})}$ \\
\hline Periodontal probing (FMPP) $(\mathrm{mm})$ & $2.17[1.99-2.34]$ & $2.52[2.36-2.75]$ & $<0.001^{(\mathrm{c})}$ \\
\hline Clinical attachment loss CAL $(\mathrm{mm}) \geq 2 \mathrm{~mm}$ & ---- & $2.38[2.00-3.42]$ & ------- \\
\hline Decayed teeth & $2[1-3]$ & $4[3-5]$ & $<0.001^{\text {(c) }}$ \\
\hline Yeast CFU $(/ \mathrm{mL})$ & $85[46-123]$ & $197[123-274]$ & $<0.001^{(\mathrm{c})}$ \\
\hline
\end{tabular}

All clinical variables are expressed as median and quartiles, except gender and age. (a) Chi2; (b) Student's $t$; (c) Mann-Whitney.

TABle 2: The presence of Candida albicans and Candida dubliniensis according to sex, age, and clinical variables.

\begin{tabular}{|c|c|c|c|c|c|c|}
\hline & \multicolumn{2}{|c|}{ Candida albicans } & \multirow{2}{*}{$P$ value } & \multicolumn{2}{|c|}{ Candida dubliniensis } & \multirow{2}{*}{$P$ value } \\
\hline & Absence $N=341$ & Presence $N=85$ & & Absence $N=409$ & Presence $N=17$ & \\
\hline \multirow{2}{*}{ Gender $(n, \%)$} & $154(45.2 \%)$ & $38(44.7 \%)$ & $0.940^{(a)}$ & $186(45.5 \%)$ & $6(35.3 \%)$ & $0.408^{(a)}$ \\
\hline & $187(54.8 \%)$ & $47(55.3 \%)$ & & $223(54.5 \%)$ & $11(64.7 \%)$ & \\
\hline Age (years) $($ mean $\pm S D)$ & $18.26 \pm 3.17$ & $17.95 \pm 3.34$ & $0.437^{(\mathrm{b})}$ & $18.16 \pm 3.20$ & $18.94 \pm 3.19$ & $0.328^{(\mathrm{b})}$ \\
\hline $\begin{array}{l}\text { Periodontal probing }(\mathrm{PP}) \\
(\mathrm{mm})\end{array}$ & $2.20[2.00-2.40]$ & $2.29[2.09-2.53]$ & $0.004^{(\mathrm{c})}$ & $2.22[2.01-2.41]$ & $2.28[2.04-2.48]$ & $0.364^{(\mathrm{c})}$ \\
\hline PPD $>3 \mathrm{~mm}$ pocket depth & $4.43[4.25-5.00]$ & $4.45[4.20-4.73]$ & $0.720^{\text {(c) }}$ & $4.40[4.24-4.78]$ & $4.50[4.48-5.50]$ & $0.990^{(\mathrm{c})}$ \\
\hline $\begin{array}{l}\text { Clinical attachment loss } \\
\text { CAL }(\mathrm{mm}) \geq 2 \mathrm{~mm}\end{array}$ & $2.46[2.00-3.60]$ & $2.21[2.00-3.00]$ & $0.481^{(c)}$ & $2.38[2.00-3.08]$ & $2.78[2.00-5.07]$ & $0.415^{(\mathrm{c})}$ \\
\hline Decayed teeth & $2[1-3]$ & $2[1-4]$ & $0.004^{(\mathrm{c})}$ & $2[1-3]$ & $2[1-3]$ & $0.701^{(\mathrm{c})}$ \\
\hline Presence of periodontitis & $43(63 \%)$ & $25(37 \%)$ & $<0.001^{(\mathrm{a})}$ & $62(91 \%)$ & $6(9 \%)$ & $0.026^{(\mathrm{a})}$ \\
\hline
\end{tabular}

All clinical variables are expressed as median and quartiles (nonparametric distributions), except gender and age. (a) Chi2; (b) Student's t; (c) Mann-Whitney.

TABLE 3: Comparison of age, clinical variables, and presence of Candida albicans and Candida dubliniensis according to stages in periodontitis group.

\begin{tabular}{|c|c|c|c|c|}
\hline & $\begin{array}{l}\text { Subgroup 1: Stage I, } \\
\qquad N=27\end{array}$ & $\begin{array}{l}\text { Subgroup 2: Stage II, } \\
\qquad N=33\end{array}$ & $\begin{array}{l}\text { Subgroup 3: Stage III, } \\
\qquad N=8\end{array}$ & $P$ value \\
\hline Age (years) $($ mean $\pm S D)$ & $19.66 \pm 2.33$ & $20.33 \pm 2.20$ & $21.62 \pm 2.32$ & $0.101^{\text {(a) }}$ \\
\hline Periodontal probing $(\mathrm{PP})(\mathrm{mm})$ & $2.54[2.32-2.72]$ & $2.45[2.36-2.72]$ & $3.90[2.58-5.73]$ & $0.001^{(b)}$ \\
\hline Pocket depth $>3 \mathrm{~mm}$ & $4.33[4.20-4.50]$ & $4.40[4.25-4.76]$ & $6.00[4.78-8.37]$ & $<0.001^{\text {(b) }}$ \\
\hline $\begin{array}{l}\text { Clinical attachment loss CAL }(\mathrm{mm}) \geq \\
2 \mathrm{~mm}\end{array}$ & $2.00[2.00-2.00]$ & $2.88[2.44-3.50]$ & $6.03[4.45-7.75]$ & $<0.001^{\text {(b) }}$ \\
\hline Presence of Candida albicans & $11(44 \%)$ & $11(44 \%)$ & $3(12 \%)$ & $0.838^{(\mathrm{c})}$ \\
\hline Presence of Candida dubliniensis & $3(50 \%)$ & $2(33 \%)$ & $1(17 \%)$ & $0.732^{(\mathrm{c})}$ \\
\hline
\end{tabular}

All clinical variables are expressed as median and quartiles (nonparametric distributions), except gender and age. (a) ANOVA, (b) Kruskal-Wallis, and (c) Chi2.

TABLE 4: Comparison of age, clinical variables, and presence of Candida albicans and Candida dubliniensis according to grades in periodontitis group.

\begin{tabular}{|c|c|c|c|}
\hline & Grade $\mathrm{B}, N=45$ & Grade C, $N=23$ & $P$ value \\
\hline Age (years) $($ mean $\pm \mathrm{SD})$ & $20.00 \pm 2.13$ & $20.65 \pm 2.63$ & $0.275^{\text {(a) }}$ \\
\hline Periodontal probing (PP) (mm) & $2.48[2.32-2.69]$ & $2.66[2.45-3.44]$ & $0.002^{(b)}$ \\
\hline Pocket depth $>3 \mathrm{~mm}$ & $4.33[4.19-4.50]$ & $5.00[4.44-5.29]$ & $<0.001^{\text {(b) }}$ \\
\hline Clinical attachment loss CAL $(\mathrm{mm}) \geq 2 \mathrm{~mm}$ & $2.00[2.00-2.46]$ & $3.86[2.60-5.00]$ & $<0.001$ (b) \\
\hline Presence of Candida albicans & $17(68 \%)$ & $08(32 \%)$ & $0.809^{(\mathrm{c})}$ \\
\hline Presence of Candida dubliniensis & $4(67 \%)$ & $2(33 \%)$ & $0.979^{(\mathrm{c})}$ \\
\hline
\end{tabular}

All clinical variables are expressed as median and quartiles (nonparametric distributions), except gender and age. (a) Student's $t$, (b) Mann-Whitney, and (c) Chi2. 
CAL was $5 \mathrm{~mm}$, whereas the chosen threshold of CAL in our study was $2 \mathrm{~mm}$ to avoid underestimating the initial lesions of periodontitis. Data on the prevalence and determinants of periodontal disease, particularly in adolescents, helps the practitioner in diagnosing and managing cases before the onset of irreversible periodontal disease states.

Periodontal diseases are associated with several microorganisms of the subgingival plaque such as Porphyromonas gingivalis (P.g.) Actinobacillus actinomycetemcomitans (A.a.), Prevotella intermedia, and Fusobacterium nucleatum [25], but the role of oral yeasts in these diseases is not known until now. Compared to subjects with healthy periodontium, studies have reported an increased prevalence of Candida in periodontal pockets, particularly Candida albicans, in patients with periodontitis $[6,26]$. However, these studies have been limited by microbial identification methods and/or small sample size.

Cuesta et al. [27] reported a value of $13 \%$ of the genus Candida of which $76.2 \%$ were Candida albicans in a population of 82 with 56 chronic periodontitis, which is confirmed by several studies and which also reported that Candida albicans was the most frequent species in periodontal pockets by $21.7 \%$ to $24.4 \%[12,28]$.

According to previous studies, the distribution of Candida albicans isolates from the subgingival biofilm according to periodontal health status varied between 9,5\% and $40 \%$ in subjects with chronic periodontitis, whereas in healthy subjects, the presence of Candida albicans was estimated between $3.8 \%$ and $36 \%$ [11, 26, 28-32] and $20 \%$ in patients with aggressive periodontitis [6]. In our study, Candida albicans was recovered in the periodontal pockets of $25(37 \%)$ subjects with periodontitis versus $17 \%$ without periodontitis (Table 2).

The second species identified was Candida dubliniensis; this is a recently identified opportunistic pathogenic yeast associated with oral candidiasis, particularly in HIV-infected individuals. Few epidemiological studies have evaluated the prevalence of colonization by this species in periodontal pockets and its possible role in the oral environment of healthy and periodontitis patients [33]. A few studies have reported Candida dubliniensis colonization in the subgingival plaque of immunocompetent subjects with periodontal disease, with a prevalence of $4.4 \%$ [11] to $4.6 \%$ [28].

In this study, Candida dubliniensis colonizes periodontal pockets of 17 adolescents which represent $3.9 \%$ of the total population (Table 2).

Previous studies showed the presence of Candida $d u b$ liniensis between $2.5 \%$ and $7.7 \%$ in subjects with chronic periodontitis $[6,11,28] 2.5 \%[26]$ and less frequently in healthy individuals $(2.5 \%)[11,12]$.

Jewtuchowicz et al. in 2009 [28] found that there was no statistically significant difference $(P>0.05)$ when studying Candida dubliniensis colonization in subgingival sites in immunocompetent individuals with periodontal disease and those with good periodontal health, while other authors did not find Candida dubliniensis in the gingival sulcus in healthy individuals $[6,26,29,34]$ nor in patients with chronic [29] and aggressive [6] periodontitis.
In our study, Candida dubliniensis was recovered from the periodontal pockets of 6 subjects with periodontitis (9\%) versus $3 \%$ who did not have the disease (11 subjects) (Table 2).

We consider that these percentage differences could be related to geographical differences, as different etiologies of some fungal infections have been recognized according to geographical location and infectious niche $[35,36]$.

To analyze the degree of Candida colonization in the subgingival sites, the CFU variable was compared between the two groups. In general terms, the results showed a different distribution between the healthy and the sick; thus the sick group had a higher average CFU (197 [123-274] $\mathrm{CFU} / \mathrm{mL}$ ) compared to the control group (85 [46-123] CFU/ $\mathrm{mL}$ ) with a statistically significant difference between the two groups $(P<0.001)$ (Table 1$)$; these results are corroborated by those of Ursula et al. in 2008 [6], while Janire et al. in 2018 found this difference between the groups but without statistical significance since the $P=0.3$ [32].

Researchers are interested in identifying this species in $\mathrm{HIV}$-infected persons, especially in cases of oropharyngeal candidiasis, and for this reason, it can be concluded that it is an important human pathogen, although it is a rare constituent of the microbial flora of the normal human organism [34, 37], which is also confirmed by our study (rare species in patients with healthy and diseased periodontium) (Table 2).

Among the 426 subjects, women and men with Candida albicans germ were, respectively, $19 \%$ and $20 \%$ in this study and therefore the presence of this species is not related to sex $(P=0.940)$ (Table 2$)$, a result confirmed by several studies $[10,26,38]$, although Reynaud in 2001 shows a slight difference in prevalence in women $(20.3 \%)$ compared to men $(8.2 \%)[10]$.

When analyzing periodontitis according to the severity and probable rate of progression, we noticed that there was no significant difference between stages or grades subgroups of periodontitis (Tables 3 and 4). However, we assume that the small sizes of subgroups may interfere with the results that we found. Canabaro et al. 2013 reported that Candida albicans was associated with severity of periodontitis [26].

In the present study, the median of decayed teeth was 2.0 [1.0-3.0], and when comparing this variable with the presence or absence of Candida albicans, we found a statistically significant difference $(P<0.05)$ (Table 2$)$. This is corroborated with results which showed the high presence of Candida albicans in children with caries [39]. We also noticed that in periodontitis group, the number of decayed teeth was significant in comparison with periodontally healthy subjects.

This important observation was recently discussed by Nigrini et al. 2019, who reported that the physical interaction between Candida and some oral bacteria helps coadhesion within biofilms and promotes development chemical interactions that enhance the coexistence of this species [40].

The comparison of the average of decayed teeth of the students in our study between the two groups showed a statistically significant difference $(P<0.05)$, which can justify the relationship of the appearance of the two diseases. 
The biofilm, where all the metabolic activities of these microorganisms take place, is one of the factors associated with the pathological progression and severity of oral diseases (caries and periodontitis), in addition to genetic factors, oral hygiene, individual and cultural behaviors and mode of nutrition, and factors related to host health, such as immunity and salivary secretion. In this regard, it can be concluded that the processes that lead to the appearance of caries and periodontitis are similar.

The microbial etiology of caries is related to polymicrobial infection of teeth and bacterial-fungal interactions. Several previous studies have explored the cariogenic role of Candida species and found that Candida albicans promotes biofilm formation and accumulation [41-43].

A synergistic interaction between Candida albicans and oral bacteria promoting the virulence of polymicrobial biofilms was reported $[44,45]$. Indeed, regarding the specific case of periodontitis, the consumption of oxygen by Candida albicans seems to create an oxygen tension that helps $P$. gingivalis growth [46] and support $P$. gingivalis ability to invade host cells [47]. However, A. actinomycetemcomitans, bacteria strongly associated with periodontitis in teenagers and young adults, seems to inhibit fungal biofilm development [48].

Thus, the potential role of Candida albicans in periodontitis pathogenesis is very complex. More studies on biofilm associated with different forms of periodontitis are necessary. It is also important to assess the coexistence of periodontitis and caries and the associated biofilms.

\section{Conclusion}

In this study Candida albicans was more frequently isolated in periodontal pockets than Candida dubliniensis. However, further studies are required to understand the potential role of this yeast in the biofilm associated with periodontal disease. In this study, the number of decayed teeth was statistically significant in periodontitis patients. This observation pointed out the complexity of biofilms associated to periodontitis and caries, in which Candida albicans may have an influence on microbial colonization and coexistence.

\section{Data Availability}

All data are included within the manuscript.

\section{Conflicts of Interest}

The authors declare no conflicts of interest.

\section{Authors' Contributions}

B. Jabri was responsible for conceptualization, data curation, formal analysis, investigation, methodology, and writing of the original draft. M. Iken was responsible for conceptualization, data curation, formal analysis, investigation, methodology, software, supervision, review, and editing. S. Ait Oamar was responsible for conceptualization, data curation, investigation, methodology, review, and editing. S. Rida was responsible for funding acquisition, project administration, resources, and review of the final manuscript. A. Bouziane was responsible for conceptualization, data curation, formal analysis, methodology, review, and editing. Ok. Ennibi was responsible for conceptualization, data curation, formal analysis, funding acquisition, investigation, methodology, project administration, resources, supervision, validation, visualization, review, and editing.

\section{Acknowledgments}

This work was supported by Centre National pour la Recherche Scientifique et Technique, Morocco (CNRST), under Grant no. PPR16/2016. The authors would like to thank Pr Lmimouni B., who provided us with the Laboratory of Parasitology and Mycology facilities, Mohammed V Military Training Hospital, Rabat, Morocco, and they would like to acknowledge the precious help of Dr. Iken M.

\section{References}

[1] P. I. Eke, G. Thornton-Evans, B. Dye, and R. Genco, "Advances in surveillance of periodontitis: the Centers for Disease Control and Prevention periodontal disease surveillance project," Journal of Periodontology, vol. 83, no. 11, pp. 1337-1342, 2012.

[2] J. Meyle and I. Chapple, "Molecular aspects of the pathogenesis of periodontitis," Periodontol 2000, vol. 69, no. 1, pp. 7-17, 2015.

[3] Y. W. Han and X. Wang, "Mobile microbiome," Journal of Dental Research, vol. 92, no. 6, pp. 485-491, 2013.

[4] D. Zuanazzi, R. Souto, M. B. A. Mattos et al., "Prevalence of potential bacterial respiratory pathogens in the oral cavity of hospitalised individuals," Archives of Oral Biology, vol. 55, no. 1, pp. 21-28, 2010.

[5] M. S. Tonetti and N. Claffey, "Advances in the progression of periodontitis and proposal of definitions of a periodontitis case and disease progression for use in risk factor research. Group C consensus report of the 5th European Workshop in Periodontology," Journal of Clinical Periodontology, vol. 32, no. s6, pp. 210-213, 2005.

[6] B. Urzúa, G. Hermosilla, J. Gamonal et al., "Yeast diversity in the oral microbiota of subjects with periodontitis:Candida albicansandCandida dubliniensiscolonize the periodontal pockets," Medical Mycology, vol. 46, no. 8, pp. 783-793, 2008.

[7] K. Yuan, C.-j. Chang, P.-c. Hsu, H. S. Sun, C.-c. Tseng, and J.-r. Wang, "Detection of putative periodontal pathogens in non-insulin-dependent diabetes mellitus and non-diabetes mellitus by polymerase chain reaction," Journal of Periodontal Research, vol. 36, no. 1, pp. 18-24, 2001.

[8] O. Gudlaugsson, S. Gillespie, K. Lee et al., "Attributable mortality of nosocomial candidemia, revisited," Clinical Infectious Diseases, vol. 37, no. 9, pp. 1172-1177, 2003.

[9] M. Patel, M. Coogan, and J. S. Galpin, "Periodontal pathogens in subgingival plaque of HIV-positive subjects with chronic periodontitis," Oral Microbiology and Immunology, vol. 18, no. 3, pp. 199-201, 2003.

[10] A. H. Reynaud, B. Nygaard-Østby, G.-K. Bøygard, E. R. Eribe, I. Olsen, and P. Gjermo, "Yeasts in periodontal pockets," Journal of Clinical Periodontology, vol. 28, no. 9, pp. 860-864, 2001.

[11] V. M. Jewtuchowicz, M. I. Brusca, M. T. Mujica et al., "Subgingival distribution of yeast and their antifungal susceptibility in immunocompetent subjects with and without 
dental devices," Acta Odontologica Latinoamericana: AOL, vol. 20, no. 1, pp. 17-22, 2007.

[12] V. M. Jewtuchowicz, M. T. Mujica, M. I. Brusca et al., "Phenotypic and genotypic identification ofCandida dubliniensisfrom subgingival sites in immunocompetent subjects in Argentina," Oral Microbiology and Immunology, vol. 23, no. 6, pp. 505-509, 2008.

[13] M. Khlif, A. Sellami, H. Sellami, F. Makni, and A. Ayadi, "Candida dubliniensis: identification methods and epidemiologic implication," Pathologie Biologie, vol. 59, no. 3, pp. 166-172, 2008.

[14] P. N. Papapanou and C. Susin, "Periodontitis epidemiology: is periodontitis under-recognized, over-diagnosed, or both?" Periodontol 2000, vol. 75, no. 1, pp. 45-51, 2017.

[15] M. S. Tonetti, S. Jepsen, L. Jin, and J. Otomo-Corgel, "Impact of the global burden of periodontal diseases on health, nutrition and wellbeing of mankind: a call for global action," Journal of Clinical Periodontology, vol. 44, no. 5, pp. 456-462, 2017.

[16] J. C. O. Sardi, C. Duque, J. F. Höfling, and R. B. Gonçalves, "Genetic and phenotypic evaluation ofCandida albicansstrains isolated from subgingival biofilm of diabetic patients with chronic periodontitis," Medical Mycology, vol. 50, no. 5, pp. 467-475, 2012.

[17] R. Lamichhane, K. Boaz, S. Natarajan, and M. Shrestha, "Assessment of candidal carriage in patients with type II diabetes mellitus," Journal of Pathology of Nepal, vol. 5, no. 9, pp. 733-738, 2015.

[18] M. Whiteway and C. Bachewich, "Morphogenesis in Candida albicans," Annual Review of Microbiology, vol. 61, no. 1, pp. 529-553, 2007.

[19] V. Baelum, S. Pisuithanakan, R. Teanpaisan et al., "Periodontal conditions among adults in Southern Thailand," Journal of Periodontal Research, vol. 38, no. 2, pp. 156-163, 2003.

[20] J. König, B. Holtfreter, and T. Kocher, "Periodontal health in Europe: future trends based on treatment needs and the provision of periodontal services-position paper 1," European Journal of Dental Education, vol. 14, pp. 4-24, 2010.

[21] B. Holtfreter, C. Schwahn, R. Biffar, and T. Kocher, "Epidemiology of periodontal diseases in the study of health in pomerania," Journal of Clinical Periodontology, vol. 36, no. 2, pp. 114-123, 2009.

[22] J. M. Albandar, M. B. Muranga, and T. E. Rams, "Prevalence of aggressive periodontitis in school attendees in Uganda," Journal of Clinical Periodontology, vol. 29, no. 9, pp. 823-831, 2002.

[23] A. Elamin, J. M. Albandar, K. Poulsen, R. W. Ali, and V. Bakken, "Prevalence of Aggregatibacter actinomycetemcomitans in Sudanese patients with aggressive periodontitis: a case-control study," Journal of Periodontal Research, vol. 46, no. 3, pp. 285-291, 2011.

[24] J. Kissa, S. Chemlali, B. El Houari et al., "Aggressive and chronic periodontitis in a population of Moroccan school students," Journal of Clinical Periodontology, vol. 43, no. 11, pp. 934-939, 2016.

[25] G. Hajishengallis and R. J. Lamont, "Beyond the red complex and into more complexity: the polymicrobial synergy and dysbiosis (PSD) model of periodontal disease etiology," Molecular Oral Microbiology, vol. 27, no. 6, pp. 409-419, 2012.

[26] A. Canabarro, C. Valle, M. R. Farias, F. B. Santos, M. Lazera, and B. Wanke, "Association of subgingival colonization of Candida albicans and other yeasts with severity of chronic periodontitis," Journal of Periodontal Research, vol. 48, no. 4, pp. 428-432, 2013.

[27] A. I. Cuesta, V. Jewtuchowicz, M. I. Brusca, M. L. Nastri, and A. C. Rosa, "Prevalence of Staphylococcus spp and Candida spp in the oral cavity and periodontal pockets of periodontal disease patients," Acta Odontologica Latinoamericana: AOL, vol. 23, no. 1, pp. 20-26, 2010.

[28] V. Jewtuchowicz, M. Mujica, M. Malzone, A. Cuesta, M. Nastri, and C. Iovannitti, "Genetic relatedness of subgingival and buccal Candida dubliniensis isolates in immunocompetent subjects assessed by RAPD-PCR," Journal of Oral Microbiology, vol. 1, no. 1, p. 2003, 2009.

[29] S. Matic Petrovic, M. Radunovic, M. Barac et al., "Subgingival areas as potential reservoirs of different Candida spp in type 2 diabetes patients and healthy subjects," PLoS One, vol. 14, no. 1, Article ID e0210527, 2019.

[30] A. P. Vieira Colombo, C. B. Magalhães, F. A. R. R. Hartenbach, R. Martins do Souto, and C. Maciel da Silva-Boghossian, "Periodontal-disease-associated biofilm: a reservoir for pathogens of medical importance," Microbial Pathogenesis, vol. 94, pp. 27-34, 2016.

[31] A. Järvensivu, J. Hietanen, R. Rautemaa, T. Sorsa, and M. Richardson, "Candida yeasts in chronic periodontitis tissues and subgingival microbial biofilms in vivo," Oral Diseases, vol. 10, no. 2, pp. 106-112, 2004.

[32] J. De-La-Torre, G. Quindós, C. Marcos-Arias et al., "Oral Candida colonization in patients with chronic periodontitis. Is there any relationship?" Revista Iberoamericana De Micologia, vol. 35, no. 3, pp. 134-139, 2018.

[33] D. Sullivan and D. Coleman, "Candida dubliniensis: characteristics and identification," Journal of Clinical Microbiology, vol. 36, no. 2, pp. 329-334, 1998.

[34] M. B. Portela, I. P. R. Souza, E. M. M. B. Costa, A. N. Hagler, R. M. A. Soares, and A. L. S. Santos, "Differential recovery of Candida species from subgingival sites in human immunodeficiency virus-positive and healthy children from Rio de Janeiro, Brazil," Journal of Clinical Microbiology, vol. 42, no. 12, pp. 5925-5927, 2004.

[35] Y. Kamikawa, D. Hirabayashi, T. Nagayama et al., "In vitro antifungal activity against OralCandidaSpecies using a denture base coated with silver nanoparticles," Journal of Nanomaterials, vol. 2014, Article ID 780410, 6 pages, 2014.

[36] B. P. Krom, S. Kidwai, and J. M. Ten Cate, "Candida and other fungal species," Journal of Dental Research, vol. 93, no. 5, pp. 445-451, 2014.

[37] E. P. Milan, P. de Laet Sant' Ana, A. S. De Azevedo Melo et al., "Multicenter prospective surveillance of oral Candida dubliniensis among adult Brazilian human immunodeficiency virus-positive and AIDS patients," Diagnostic Microbiology and Infectious Disease, vol. 41, no. 1-2, pp. 29-35, 2001.

[38] L. M. Barros, M. F. G. Boriollo, A. C. B. A. Alves, M. I. Klein, R. B. Gonçalves, and J. F. Höfling, "Genetic diversity and exoenzyme activities of Candida albicans and Candida dubliniensis isolated from the oral cavity of Brazilian periodontal patients," Archives of Oral Biology, vol. 53, no. 12, pp. 1172-1178, 2008.

[39] D. D. Duangthip, "Early childhood caries and candida albicans," Evidence-Based Dentistry, vol. 19, no. 4, pp. 100-101, 2018.

[40] T. d. C. Negrini, H. Koo, and R. A. Arthur, "Candida-bacterial biofilms and host-microbe interactions in oral diseases," Oral Mucosal Immunity and Microbiome, vol. 1197, pp. 119-141, 2019. 
[41] T. Klinke, B. Guggenheim, W. Klimm, and T. Thurnheer, "Dental caries in rats associated with Candida albicans," Caries Research, vol. 45, no. 2, pp. 100-106, 2011.

[42] M. Falsetta, M. Klein, P. Colonne, K. Scott-Anne, S. Gregoire, and C. Pai, "Symbiotic relationship between Streptococcus mutans and Candida albicans synergizes virulence of plaque biofilms in vivo," Infection and Immunity, vol. 82, no. 5, pp. 1968-1981, 2014.

[43] H. Sztajer, S. P. Szafranski, J. Tomasch et al., "Cross-feeding and interkingdom communication in dual-species biofilms of Streptococcus mutans and Candida albicans," The ISME Journal, vol. 8, no. 11, pp. 2256-2271, 2014.

[44] H. Koo, D. R. Andes, and D. J. Krysan, "Candida-streptococcal interactions in biofilm-associated oral diseases," PLoS Pathogens, vol. 14, no. 12, Article ID e1007342, 2018.

[45] D. Montelongo-Jauregui and J. Lopez-Ribot, "Candida interactions with the oral bacterial microbiota," Journal of Fungi, vol. 4, no. 4, p. 122, 2018.

[46] M. N. Sztukowska, L. C. Dutton, C. Delaney et al., "Community development between Porphyromonas gingivalis and Candida albicans mediated by InlJ and Als3," mBio, vol. 9, no. 2, 2018.

[47] R. Tamai, M. Sugamata, and Y. Kiyoura, "Candida albicans enhances invasion of human gingival epithelial cells and gingival fibroblasts by Porphyromonas gingivalis," Microbial Pathogenesis, vol. 51, no. 4, pp. 250-254, 2011.

[48] E. W. Bachtiar, B. M. Bachtiar, L. M. Jarosz et al., "AI-2 of Aggregatibacter actinomycetemcomitans inhibits Candida albicans biofilm formation," Frontiers in Cellular and Infection Microbiology, vol. 4, p. 94, 2014. 